\title{
A literature review on lactopontin and its roles in early life
}

\author{
Qiong Jia ${ }^{1 \# \wedge}$, Yiran Wang ${ }^{2 \#}$, Jing $\mathrm{Zhu}^{2} \wedge$, Huanling $\mathrm{Yu}^{3}$, Xiaomei Tong ${ }^{1 \wedge}$ \\ ${ }^{1}$ Department of Pediatrics, Peking University Third Hospital, Beijing, China; ${ }^{2}$ Department of Nutritional and Functional Assessment, Beijing \\ Institute of Nutritional Resources, Beijing, China; ${ }^{3}$ Department of Nutrition and Food Hygiene, School of Public Health, Capital Medical \\ University, Beijing, China \\ Contributions: (I) Conception and design: J Zhu, X Tong; (II) Administrative support: J Zhu, H Yu, X Tong; (III) Provision of study materials or \\ patients: None; (IV) Collection and assembly of data: Q Jia, Y Wang; (V) Data analysis and interpretation: Q Jia, Y Wang; (VI) Manuscript writing: \\ All authors; (VII) Final approval of manuscript: All authors. \\ \#These authors contributed equally to this work. \\ Correspondence to: Jing Zhu. Beijing Institute of Nutritional Resources, Beijing, China. Email: jingzhu.nutri@outlook.com; Huanling Yu. Department \\ of Nutrition and Food Hygiene, School of Public Health, Capital Medical University, Beijing, China. Email: yuhlzjl@ccmu.edu.cn; Xiaomei Tong. \\ Department of Pediatrics, Peking University Third Hospital, Beijing, China. Email: tongxm2007@126.com.
}

Objective: Our study aims to review the functions and possible mechanisms of lactopontin (LPN) in early life.

Background: Human milk proteins provide a variety of protection and health benefits in early life. One of these multifunctional proteins is LPN, which is osteopontin (OPN) derived from milk.

Methods: Information used to write this paper was collected from Uniprot, PubMed, and Google Scholar, including in vitro, in vivo, and clinical studies.

Conclusions: LPN is a highly phosphorylated, $O$-glycosylated acidic protein and a unique type of OPN, as it presents at the highest concentration and a higher degree of posttranslational modifications (PTMs) in human milk than other tissues and excretions. LPN is present in milk and the intestinal tracts of infants after consumption as a mixture of intact protein and peptides, which can bind diverse integrin and receptors in the target cell and drive downstream signaling pathways. LPN is found to play important roles in developing the immune, intestinal and nervous systems in early life. Moreover, LPN has also shown to support preterm infants' health when they are especially vulnerable after delivery via animal studies. Additionally, LPN can form protein complex with another milk bioactive protein, lactoferrin (LF), to withstand proteolysis and perform more efficient biological activity. Therefore, LPN showed great potential for early life while more clinical trials and evidence are still emergying.

Keywords: Lactopontin (LPN); milk osteopontin; immune regulation; gut development; neurodevelopment

Submitted Jun 14, 2021. Accepted for publication Jul 14, 2021.

doi: $10.21037 / \mathrm{tp}-21-293$

View this article at: https://dx.doi.org/10.21037/tp-21-293

\section{Introduction}

The mammary glands of female mammals secrete milk to nourish their offspring during early life after birth. Milk is a natural source of nutrients and bioactive proteins necessary for the health and growth of infants. Many studies have identified the short and long-term benefits for both maternal health and infant development (1). Proteins in milk are increasingly appreciated for their bioactive functions rather than a source of essential amino acids. In recent years, osteopontin (OPN) in milk has attracted much attention. OPN was initially found in bone tissue and is

^ ORCID: Qiong Jia, 0000-0002-8323-6072; Jing Zhu, 0000-0001-8451-9173; Xiaomei Tong, 0000-0003-4477-4123. 


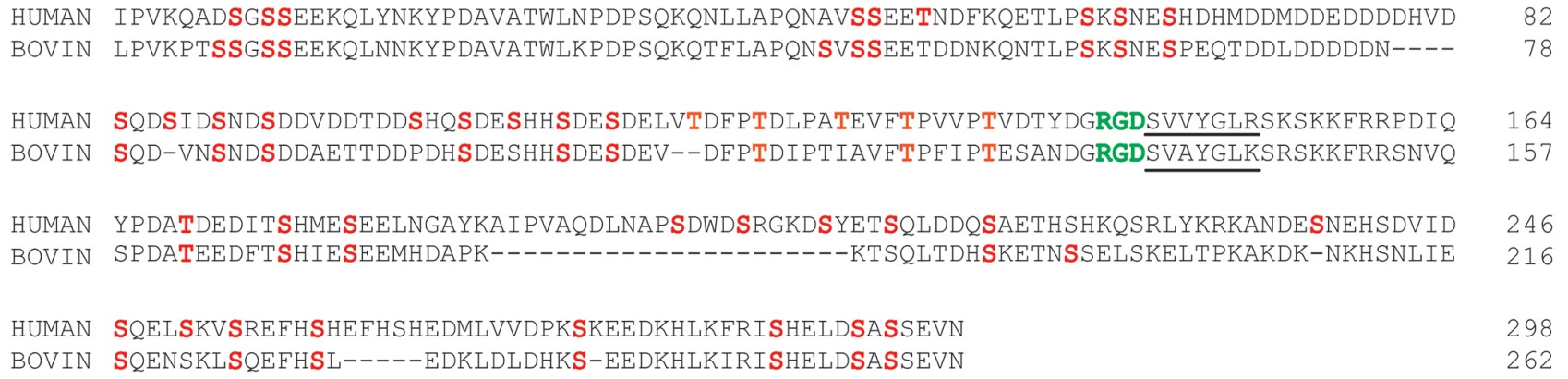

HUMAN SQDSIDSNDSDDVDDTDDSHQSDESHHSDESDELVTDFPTDLPATEVFTPVVPTVDTYDGRGDSVVYGLRSKSKKFRRPDIQ

Figure 1 Alignment of human and bovine LPN sequences. Broken lines indicate introduced gaps. Residues for phosphorylation and glycosylation are highlighted in red and orange, respectively. RGD (Arg-Gly-Asp) motif is colored in green, and the cryptic sequence is underlined. LPN, lactopontin.

considered an important bone matrix protein $(2,3)$.

Further studies revealed that OPN could be synthesized or secreted by various tissues and cells in the body. OPN is present in two forms: intracellular OPN found in immune cells (4) and secreted OPN present in body fluid, such as milk, urine, blood, saliva, and bile (5). OPN in milk, also known as lactopontin (LPN) $(6,7)$, is a remarkable type of $\mathrm{OPN}$ as it is found in higher concentrations in human milk than OPN in other tissues and excretions (8), it has a higher degree of posttranslational modifications (PTMs) (9-11), and the mother secretes it but ingested by the offspring as food and plays important roles in the infant's growth and health (3).

Microarray analyses of cells in human milk revealed that the secreted phosphoprotein 1 (SPP1) gene which code LPN is highly expressed throughout the lactational stages (12). The average concentration of LPN in human milk $(138 \mathrm{mg} / \mathrm{L})$ is about 8 times higher than in bovine milk (18 mg/L) (3). One study recruited 629 lactating mothers (mean age 31.4 years) from four countriesChina, Japan, Korea, and Denmark—and collected 829 breast milk samples (13). The mean LPN in the breast milk of Chinese mothers 4.3 weeks postpartum $(266.2 \mathrm{mg} / \mathrm{L})$ was significantly higher than that of Korean mothers at 3.9 weeks postpartum $(216.2 \mathrm{mg} / \mathrm{L})$, Japanese mothers 9.1 weeks postpartum $(185 \mathrm{mg} / \mathrm{L})$, and Danish mothers 17.4 weeks postpartum $(99.7 \mathrm{mg} / \mathrm{L})$. Moreover, the proportion of LPN in total milk proteins was also higher in Chinese mothers $(2.7 \%)$ than in Korean mothers (1.8\%), Japanese mothers (2.4\%), and Danish mothers (1.3\%) (13). Another study examined the changes in LPN in the milk of 12 mothers of full-term infants during the 12 months postpartum and found that LPN concentration was highest in colostrum $(178.0 \pm 17.9 \mathrm{mg} / \mathrm{L})$, gradually decreased from colostrum to transitional milk to mature milk, and remained a stable concentration in late lactation (14). The higher concentration of LPN in colostrum suggests LPN may play a critical role in the early life of infants. Recent studies have reported different effects of LPN in early life by in vitro, animal studies, and clinical trials in infants. This study aims to summarize the recent findings, conduct a literature review on the functions and possible mechanisms of LPN in early life, and discuss future perspectives. We present the following article in accordance with the Narrative Review reporting checklist (available at https://dx.doi.org/10.21037/tp-21-293).

\section{Methods}

The literature that was reviewed in this paper was sourced from Uniprot, PubMed, and Google Scholar. The protein sequence and modified sites were searched in Uniprot by the gene name "SPP1" using the Swiss-Prot database. The keywords for the literature search in Pubmed and Google Scholar were "osteopontin," "lactopontin," and "milk." All articles collected for this study were published before April 30, 2021. The reference lists of relevant articles were manually searched for additional literature. Only publications in English were reviewed.

\section{Structural characteristics of LPN}

LPN is a highly phosphorylated, $O$-glycosylated acidic protein that is structurally similar among different species. For example, as shown in Figure 1, human LPN contains 298 amino acids (AAs), and bovine LPN contains 262 AAs, of which $182(61 \%)$ are identical to human LPN and 44 are highly structurally similar (8). LPN in all species 
has an integrin-binding Arg-Gly-Asp (RGD) motif and a cryptic sequence (human: SVVYGLR, bovine: SVAYGLK). Moreover, LPN is heavily modified by PTMs and heterogeneous proteolytic processing by milk enzymes.

Most of the modified residues and regulatory proteolytic cleavage sites are conserved between human and bovine LPN. Human LPN contains 36 phosphorylation sites (34 phosphoserine sites and 2 phosphothreonine sites) and 5 $O$-glycosylation sites (15). In comparison, bovine LPN has 28 phosphorylation sites (27 phosphoserine sites and 1 phosphothreonine site) and $3 \mathrm{O}$-glycosylation sites, wherein 25 phosphorylation sites and $3 O$-glycosylation sites are identical to human LPN $(2,14)$. The phosphorylation sites are arranged in clusters of three to five phosphoresidues predominantly located in the target sequence of the kinase FAM20C (16), while the RGD motif and the glycosites are not phosphorylated (10). In human LPN, approximately 25-26 phosphates are distributed over 36 potential sites $(10,11)$, while in bovine LPN, approximately 22 phosphates are distributed over 28 potential sites (17). Most glycan structures identified in human LPN have been shown to consist of the GalNAc-galactose-(GlcNAc) core-2 structure extended by fucosylated and, to a lesser degree, sialylated poly-N-acetyllactosamine units (11). The multifunctional bioactivity of LPN is associated with its binding to several integrins and CD44 receptors, which were affected by its phosphorylation and $O$-glycosylation statuses $(11,18)$.

In addition, LPN is susceptible to degradation by a variety of proteases naturally present in breast milk, such as thrombin, cathepsin D, and plasmin, producing endogenous LPN polypeptides of various lengths and sequences $(19,20)$. After entering the gastrointestinal tract of infants, LPN is further cleaved, and many peptides are produced. A study identified human milk and bovine milk peptides in gastric aspirates and showed the major cleavage sites of LPN were mostly conserved between the two species regarding hydrophobicity patterns. In bovine LPN, $\mathrm{Phe}^{38}-\mathrm{Leu}^{39}$ was the largest cleavage site which was also present in human LPN as $\mathrm{Leu}^{38}-\mathrm{Leu}^{39}$, whereas in human LPN, the largest cleavage site was $\operatorname{Thr}^{26}-\operatorname{Trp}^{27}$, which was also found in bovine LPN (20). The various peptides derived from LPN, in turn, bind to a range of cell surface integral proteins or CD44 receptors to perform a variety of physiological functions (21).

\section{Biological functions of LPN}

The levels of OPN in the cord blood and plasma of 3-month-old infants are 7-10 times higher than in adult plasma (3). The high levels of LPN in milk, especially in the colostrum, suggest that LPN may play an important role in early life when the infants are immunologically immature (12). LPN can bind and form a soluble complex with calcium as an acidic protein, thereby increasing the stability of calcium and inhibiting unintentional precipitation of amorphous calcium phosphate in milk (22). Moreover, LPN shows multifunctional bioactivities due to its integrin and receptor binding properties and initiates signaling pathways in the form of intact LPN or LPN peptides (23-25), as it is partly resistant to proteolysis and is prone to proteolytic cleavage in milk or the intestinal tract (15). A study found that the polypeptides formed by LPN usually contain RGD sequences and SVVYGLR motifs and still can bind integral proteins such as $\alpha v \beta 3$ (21) or even increase the binding abilities (8). Due to the structural similarity and conserved functional sites of LPN, the biological activity of LPN is similar across mammalian sources.

LPN has been shown to play a role in immunological development in infants, including defense of pathogenintroduced infections and regulation of immune response. An in vitro study found that the C-terminal fragment obtained by thrombin-treated bovine LPN could bind to $\alpha \mathrm{X} \beta 2$ (CD11c/CD18) integrins that were highly expressed on the surface of monocytes, mediate the chemoattraction of interleukin (IL)-1-activated human monocytes and exert a regulatory effect on macrophage chemotaxis, adhesion, infiltration, and phagocytosis (26). Moreover, LPN can bind Staphylococcus aureus in a dose-dependent manner under a $\mathrm{Ca}^{2+}$-dependent interaction (26). Another in vitro assay found that LPN promoted the migration and activation of dendritic cells (DCs), induced T cell proliferation, and promoted their progression to the Th1 phenotype by stimulating DC release (27). Mice deficient in SPP1 gene expression had an imbalanced Th1/Th2 immune response and were more susceptible to viral and bacterial infections than wild type mice (28). LPN may act at the gut mucosal surface of infants and regulate the immune response by increasing the expression of the Th1 cytokine interleukin-12 (IL-12) and interferon-gamma via integrin receptor binding, and decreasing the Th2 cytokine IL-10 via CD44 receptor binding (28). In a randomized controlled trial, 240 healthy infants were divided into four groups: standard formula group (no supplemental LPN, group F0), formula group with two doses of supplemental LPN (group F65: $65 \mathrm{mg} / \mathrm{L}$, group F130: $130 \mathrm{mg} / \mathrm{L}$ ), and exclusive breastfeeding group (BF group) from 1-6 months of age 
$(29,30)$. The study showed that LPN supplementation changed the plasma levels of several amino acids and cytokines in formula-fed infants to levels similar to those in breastfed infants, although some changes were not significant. Infants fed with standard formula had significantly higher serum levels of the tumor necrosis factor-alpha (TNF- $\alpha$ ) than infants fed formula with additional LPN, who did not differ from the breastfed group (29). The incidence and frequency of fever reported by parents were significantly higher in the F0 group than in the $\mathrm{BF}$ group, while there were no differences between the F65, F130, and BF groups (29). In addition, the peripheral blood T-cell ratio was significantly higher in the F130 group than in the F65 group and the F0 group and was not significantly different from that of the exclusively breastfed BF group (30). Thus, infants fed formula supplemented with bovine LPN showed a higher similarity to the breastfed infants than infants fed regular formula alone, which may support improved innate and adaptive immune development $(29,30)$.

In animal studies, LPN promoted small intestinal mucosal growth, improved duodenal villus thickness and crypt depth, increased small intestinal mucosal surface area, and enhanced its nutrient absorption efficiency (31). LPN has been reported to regulate small intestinal gene expression through the integrin signaling pathway and influence early-life intestinal proliferation and maturation by regulating cell migration and cell chemotaxis (31). LPN has also been found to play a role in protecting small intestinal mucosal integrity by affecting integral tight junction membrane proteins such as occludin and zonula occludens-1 in the intestinal mucosa (32). In an in vitro study, bovine LPN upregulated the secretion of IL-18 from intestinal epithelial cells (Caco-2), promoted Caco-2 differentiation, and stimulated intestinal immune function (33). In another in vitro study, human LPN and bovine LPN stimulated proliferation of human intestinal cells, and gene expression were changed for those tightly related to proliferation and immune function, such as MAPK13, CCNE1, CdGAP, CXCL10, IL6ST, and NFKB (34). In a study with newborn rhesus monkeys, feeding bovine LPN (125 mg/L) affected intestinal gene expression (35). From birth to 3 months, rhesus monkeys were fed either breast milk or normal formula with or without bovine LPN. Although no difference was observed in growth, body composition, or blood immune cells, small intestinal transcriptome revealed that while 1,017 genes were differentially expressed between the normal formula and breast milk groups, the supplementation of LPN in formula diminished the number of different genes from 1,017 to 217 (35). Bovine LPN added in drinking water $(20 \mu \mathrm{g} / \mathrm{mL})$ to dextran sulfate sodium (DSS)-treated wild-type (WT) mice were correlated with less weight loss, spleen enlargement, gut neutrophil activity, and pro-inflammatory mediators, and increased colon length and red blood cell counts compared with DSS-treated WT mice (36). In addition, LPN may alter intestinal flora and affect the content of short-chain fatty acids in piglets (37).

OPN is highly expressed in the brain in early infancy (38) and is crucial in forming myelin sheaths for normal brain development (39). Human LPN, administered orally in mouse pups, could enter the brain and increase the brain OPN protein level (40). The mice breastfed by mothers lacking LPN after knockout had reduced expression of myelin-related proteins and reduced proliferation and differentiation of NG-2 glial cells to oligodendrocytes compared with mice breastfed by wild type mothers, accompanied by reduced ERK-1/2 and P13K/Akt signaling, and memory and learning ability were found to be impaired using passive avoidance tests and rotarod experiments (40). Another animal study tested the bovine LPN on the brain and cognitive development in piglets (37). Piglets were fed a soy protein-based formula with or without bovine LPN $(250 \mathrm{~mL} / \mathrm{L})$ from birth to 34 days. A novel object recognition (NOR) test was applied to assess the cognitive development and found that the two groups of piglets did not show significant differences in behavioral tests, but the piglets in the LPN-enriched group had a shorter waiting time than those in the non-LPN-enriched group when first exploring new targets. Neuroimaging outcomes revealed the LPNenriched group had increased relative brain volumes of the corpus callosum, lateral ventricle, left and right internal capsule, left and right putamen-globus pallidus, right hippocampus, and right cortex. Diffusion tensor imaging revealed higher radial diffusivity in the corpus callosum and lower fractional anisotropy in the LPN-enriched group. The results suggest that bovine LPN promotes the development of neural structures and improves exploratory behavior (37).

Moreover, LPN for preterm infants is gaining attention. Newborn premature infants have shorter intrauterine development, poorer growth and development, immature development of vital organs such as brain, lung, and intestine, and greater susceptibility to various infectious diseases (41). The shorter the gestational age is, the worse 
the developmental status of preterm infants (42). LPN is an important component of preterm mothers' milk in the form of intact protein (43) and endogenous peptides (44). Animal studies have shown that compared with formula without LPN, the formula with supplementary LPN can reduce the rate of diarrhea in preterm pigs (45) and, while it does not affect the incidence of necrotizing enterocolitis, it can reduce the severity (46). The role of LPN in enhancing the maturation of the immune, intestinal and neurological systems in early life may be critical for preterm infants; thus future clinical studies are needed.

\section{Lactoferrin (LF)-LPN complex}

LPN can form complexes with several milk proteins; for instance, it can electrostatically bind to lactoferrin $\left(\mathrm{K}_{\mathrm{D}}=10^{-6} \mathrm{M}\right)$ or lactoperoxidase or bind immunoglobulin $M(\operatorname{IgM})$ with high affinity $\left(K_{D}=1.77 \times 10^{-7} M\right)(47)$. In these complexes, LPN may protect these immunomodulating and antimicrobial proteins from proteolysis (33) and perform more efficient biological activity than when alone $(24,48)$. The LF-LPN complex is one of the most common complexes naturally occurring in both human and bovine milk (24).

LF is an abundant and important multifunctional, nonheme iron-binding glycoprotein in breast milk (49). LF belongs to the transferrin family and has a high iron affinity $\left(\mathrm{K}_{\mathrm{D}}=10^{-22} \mathrm{M}\right)$ which is 260 times higher than serotransferrin (TF) (50). LF kills pathogenic microorganisms by competitively seizing iron to lack sufficient iron replenishment to maintain biofilm structure and consequently die (51). LF binds to lipopolysaccharide (LPS) on the cell wall of gram-negative bacteria, killing the bacteria by altering cell membrane permeability $(52,53)$. LF also has antiviral activity, preventing viruses from entering the host cell by blocking cellular receptors or directly binding to the virion (54). In addition, LF can recognize LF receptors on the surface of immune cells and affect immunity by promoting immune cell maturation, stimulating immune response, and immunomodulation (55). An in vitro experiment on Caco-2 revealed that LF had different functions according to its concentrations. High concentrations of LF $(1-1,000 \mu \mathrm{g} / \mathrm{mL})$ stimulated intestinal cell proliferation, while lower concentrations of LF $(1-1,000 \mu \mathrm{g} / \mathrm{mL})$ enhanced intestinal cell differentiation. LF was observed in higher concentrations during early lactation and lower concentrations during late lactation, which matched the intestinal development of the infants (56).
Since LPN and LF have opposite charges, multiple positively charged LFs can form LF-LPN complexes with one negatively charged LPN. Complexes with an LF to LPN molar ratio of 3:1 were the most active (24). The human LF-LPN complex behaved more stably than LF and LPN monomers in vitro digestion assays, and the complex was also more readily taken up by small intestinal cells than monomers, with the bovine LF-LPN complex showing similar properties (24). Another study showed that an LF-LPN complex formed by iron-free LF (apo-LF) and calcium-saturated LPN (holo-LPN) had the strongest proliferative effect on intestinal epithelial cells compared to an LF-LPN complex formed by other ion-binding types, which can withstand the action of digestive enzymes to reach the intestine and exert intestinal proliferation by activating the PI3K/Akt signaling pathway (48).

\section{Limitations to the research reviewed}

LPN is highly concentrated in human milk and is important for early infancy. However, most of the research reviewed in this article concerning supplemented LPN was in vitro or in vivo, and only one clinical trial in Chinese infants was reported. More clinical trials are needed, and systematic reviews should be done when data are efficient.

\section{Summary}

LPN is a highly concentrated and important functional component of breast milk, which plays multiple essential roles in early infancy. In vitro, in vivo, and clinical studies have revealed that LPN contributes to immune development, intestinal proliferation and maturation, and neurodevelopment. Since preterm infants are particularly immature due to shorter intrauterine development, LPN might be critical for preterm infants. As LPN naturally exists in human milk but has lower amount in bovine milk, therefore the breastfeeding infants can get enough LPN from human milk while infants feeding by infant formula may need additional supplemented LPN. However, more clinical studies are still needed to reveal the potential of LPN fully.

\section{Acknowledgments}

Funding: Beijing Municipal Natural Science Foundation No. S160004; China Postdoctoral Science Foundation No. 254063. 


\section{Footnote}

Reporting Checklist: The authors have completed the Narrative Review reporting checklist. Available at https:// dx.doi.org/10.21037/tp-21-293

Conflicts of Interest: All authors have completed the ICMJE uniform disclosure form (available at https://dx.doi. org/10.21037/tp-21-293). The authors have no conflicts of interest to declare.

Ethical Statement: The authors are accountable for all aspects of the work in ensuring that questions related to the accuracy or integrity of any part of the work are appropriately investigated and resolved.

Open Access Statement: This is an Open Access article distributed in accordance with the Creative Commons Attribution-NonCommercial-NoDerivs 4.0 International License (CC BY-NC-ND 4.0), which permits the noncommercial replication and distribution of the article with the strict proviso that no changes or edits are made and the original work is properly cited (including links to both the formal publication through the relevant DOI and the license). See: https://creativecommons.org/licenses/by-nc-nd/4.0/.

\section{References}

1. Gartner LM, Morton J, Lawrence RA, et al. Breastfeeding and the use of human milk. Pediatrics 2005;115:496-506.

2. Brown LF, Berse B, Van de Water L, et al. Expression and distribution of osteopontin in human tissues: widespread association with luminal epithelial surfaces. Mol Biol Cell 1992;3:1169-80.

3. Schack L, Lange A, Kelsen J, et al. Considerable variation in the concentration of osteopontin in human milk, bovine milk, and infant formulas. J Dairy Sci 2009;92:5378-85.

4. Rittling SR, Singh R. Osteopontin in Immune-mediated Diseases. J Dent Res 2015;94:1638-45.

5. Sodek J, Ganss B, McKee MD. Osteopontin. Crit Rev Oral Biol Med 2000;11:279-303.

6. Shiraga H, Min W, VanDusen WJ, et al. Inhibition of calcium oxalate crystal growth in vitro by uropontin: another member of the aspartic acid-rich protein superfamily. Proc Natl Acad Sci U S A 1992;89:426-30.

7. Senger DR, Perruzzi CA, Papadopoulos A, et al. Purification of a human milk protein closely similar to tumor-secreted phosphoproteins and osteopontin. Biochim
Biophys Acta 1989;996:43-8.

8. Christensen B, Sørensen ES. Structure, function and nutritional potential of milk osteopontin. International Dairy Journal 2016;57:1-6.

9. Christensen B, Petersen TE, Sørensen ES. Posttranslational modification and proteolytic processing of urinary osteopontin. Biochem J 2008;411:53-61.

10. Christensen B, Nielsen MS, Haselmann KF, et al. Post-translationally modified residues of native human osteopontin are located in clusters: identification of 36 phosphorylation and five $\mathrm{O}$-glycosylation sites and their biological implications. Biochem J 2005;390:285-92.

11. Christensen B, Kläning E, Nielsen MS, et al. C-terminal modification of osteopontin inhibits interaction with the aVß3-integrin. J Biol Chem 2012;287:3788-97.

12. Nagatomo T, Ohga S, Takada H, et al. Microarray analysis of human milk cells: persistent high expression of osteopontin during the lactation period. Clin Exp Immunol 2004;138:47-53.

13. Bruun S, Jacobsen LN, Ze X, et al. Osteopontin Levels in Human Milk Vary Across Countries and Within Lactation Period: Data From a Multicenter Study. J Pediatr Gastroenterol Nutr 2018;67:250-6.

14. Jiang R, Lönnerdal B. Osteopontin in human milk and infant formula affects infant plasma osteopontin concentrations. Pediatr Res 2019;85:502-5.

15. Jiang R, Lönnerdal B. Biological roles of milk osteopontin. Curr Opin Clin Nutr Metab Care 2016;19:214-9.

16. Tagliabracci VS, Engel JL, Wen J, et al. Secreted kinase phosphorylates extracellular proteins that regulate biomineralization. Science 2012;336:1150-3.

17. Sørensen ES, Højrup P, Petersen TE. Posttranslational modifications of bovine osteopontin: identification of twenty-eight phosphorylation and three O-glycosylation sites. Protein Sci 1995;4:2040-9.

18. Kariya Y, Kanno M, Matsumoto-Morita K, et al. Osteopontin O-glycosylation contributes to its phosphorylation and cell-adhesion properties. Biochem J 2014;463:93-102.

19. Dingess KA, van den Toorn HWP, Mank M, et al. Toward an efficient workflow for the analysis of the human milk peptidome. Anal Bioanal Chem 2019;411:1351-63.

20. Nielsen SD, Beverly RL, Underwood MA, et al. Release of functional peptides from mother's milk and fortifier proteins in the premature infant stomach. PLoS One 2018;13:e208204.

21. Zhu J, Dingess KA, Mank M, et al. Personalized Profiling Reveals Donor- and Lactation-Specific Trends 
in the Human Milk Proteome and Peptidome. J Nutr 2021;151:826-39.

22. Holt C, Lenton S, Nylander T, et al. Mineralisation of soft and hard tissues and the stability of biofluids. J Struct Biol 2014;185:383-96.

23. Christensen B, Sørensen ES. Osteopontin is highly susceptible to cleavage in bovine milk and the proteolytic fragments bind the $\alpha \mathrm{V} \beta_{3}$-integrin receptor. J Dairy Sci 2014;97:136-46.

24. Jiang R, Liu L, Du X, et al. Evaluation of Bioactivities of the Bovine Milk Lactoferrin-Osteopontin Complex in Infant Formulas. J Agric Food Chem 2020;68:6104-11.

25. Lönnerdal B, Du X, Jiang R. Biological activities of commercial bovine lactoferrin sources. Biochem Cell Biol 2021;99:35-46.

26. Schack L, Stapulionis R, Christensen B, et al. Osteopontin enhances phagocytosis through a novel osteopontin receptor, the alphaXbeta2 integrin. J Immunol 2009; 182:6943-50.

27. Renkl AC, Wussler J, Ahrens T, et al. Osteopontin functionally activates dendritic cells and induces their differentiation toward a Th1-polarizing phenotype. Blood 2005;106:946-55.

28. Ashkar S, Weber GF, Panoutsakopoulou V, et al. Eta1 (osteopontin): an early component of type-1 (cellmediated) immunity. Science 2000;287:860-4.

29. Lönnerdal B, Kvistgaard AS, Peerson JM, et al. Growth, Nutrition, and Cytokine Response of Breast-fed Infants and Infants Fed Formula With Added Bovine Osteopontin. J Pediatr Gastroenterol Nutr 2016;62:650-7.

30. West CE, Kvistgaard AS, Peerson JM, et al. Effects of osteopontin-enriched formula on lymphocyte subsets in the first 6 months of life: a randomized controlled trial. Pediatr Res 2017;82:63-71.

31. Jiang R, Lönnerdal B. Evaluation of Bioactivities of Bovine Milk Osteopontin Using a Knockout Mouse Model. J Pediatr Gastroenterol Nutr 2020;71:125-31.

32. Woo SH, Lee SH, Park JW, et al. Osteopontin Protects Colonic Mucosa from Dextran Sodium SulfateInduced Acute Colitis in Mice by Regulating Junctional Distribution of Occludin. Dig Dis Sci 2019;64:421-31.

33. Liu L, Jiang R, Lönnerdal B. Assessment of bioactivities of the human milk lactoferrin-osteopontin complex in vitro. J Nutr Biochem 2019;69:10-8.

34. Jiang R, Lonnerdal B. Human and bovine osteopontin from milk and recombinant human osteopontin may stimulate intestinal proliferation and immune functions via various mechanisms revealed by microarray analysis.
FASEB J 2013;27:45.1.

35. Donovan SM, Monaco MH, Drnevich J, et al. Bovine osteopontin modifies the intestinal transcriptome of formula-fed infant rhesus monkeys to be more similar to those that were breastfed. J Nutr 2014;144:1910-9.

36. da Silva AP, Ellen RP, Sørensen ES, et al. Osteopontin attenuation of dextran sulfate sodium-induced colitis in mice. Lab Invest 2009;89:1169-81.

37. Joung S, Fil JE, Heckmann AB, et al. Early-Life Supplementation of Bovine Milk Osteopontin Supports Neurodevelopment and Influences Exploratory Behavior. Nutrients 2020;12:2206.

38. Choi JS, Cha JH, Park HJ, et al. Transient expression of osteopontin mRNA and protein in amoeboid microglia in developing rat brain. Exp Brain Res 2004;154:275-80.

39. Selvaraju R, Bernasconi L, Losberger C, et al. Osteopontin is upregulated during in vivo demyelination and remyelination and enhances myelin formation in vitro. Mol Cell Neurosci 2004;25:707-21.

40. Jiang R, Prell C, Lönnerdal B. Milk osteopontin promotes brain development by up-regulating osteopontin in the brain in early life. FASEB J 2019;33:1681-94.

41. Collins A, Weitkamp JH, Wynn JL. Why are preterm newborns at increased risk of infection? Arch Dis Child Fetal Neonatal Ed 2018;103:F391-4.

42. Patel RM. Short- and Long-Term Outcomes for Extremely Preterm Infants. Am J Perinatol 2016;33:318-28.

43. Molinari CE, Casadio YS, Hartmann BT, et al. Proteome mapping of human skim milk proteins in term and preterm milk. J Proteome Res 2012;11:1696-714.

44. Dingess KA, de Waard M, Boeren S, et al. Human milk peptides differentiate between the preterm and term infant and across varying lactational stages. Food Funct 2017;8:3769-82.

45. Ren S, Hui Y, Goericke-Pesch S, et al. Gut and immune effects of bioactive milk factors in preterm pigs exposed to prenatal inflammation. Am J Physiol Gastrointest Liver Physiol 2019;317:G67-77.

46. Møller HK, Thymann T, Fink LN, et al. Bovine colostrum is superior to enriched formulas in stimulating intestinal function and necrotising enterocolitis resistance in preterm pigs. Br J Nutr 2011;105:44-53.

47. Azuma N, MaetaK A, Fukuchi K, et al. A rapid method for purifying osteopontin from bovine milk and interaction between osteopontin and other milk proteins. International Dairy Journal 2006;16:370-8.

48. Liu L, Jiang R, Liu J, et al. The bovine LactoferrinOsteopontin complex increases proliferation of human 
intestinal epithelial cells by activating the PI3K/Akt signaling pathway. Food Chem 2020;310:125919.

49. Demmelmair H, Prell C, Timby N, et al. Benefits of Lactoferrin, Osteopontin and Milk Fat Globule Membranes for Infants. Nutrients 2017;9:817.

50. Rosa L, Cutone A, Lepanto MS, et al. Lactoferrin: A Natural Glycoprotein Involved in Iron and Inflammatory Homeostasis. Int J Mol Sci 2017;18:1985.

51. Berlutti F, Morea C, Battistoni A, et al. Iron availability influences aggregation, biofilm, adhesion and invasion of Pseudomonas aeruginosa and Burkholderia cenocepacia. Int J Immunopathol Pharmacol 2005;18:661-70.

52. Lu J, Francis J, Doster RS, et al. Lactoferrin: A Critical Mediator of Both Host Immune Response and Antimicrobial Activity in Response to Streptococcal

Cite this article as: Jia Q, Wang Y, Zhu J, Yu H, Tong X. A literature review on lactopontin and its roles in early life. Transl Pediatr 202 1;10(7):1924-1931. doi: 10.21037/tp-21-293
Infections. ACS Infect Dis 2020;6:1615-23.

53. García-Montoya IA, Cendón TS, Arévalo-Gallegos S, et al. Lactoferrin a multiple bioactive protein: an overview. Biochim Biophys Acta 2012;1820:226-36.

54. van der Strate BW, Beljaars L, Molema G, et al. Antiviral activities of lactoferrin. Antiviral Res 2001;52:225-39.

55. Actor JK, Hwang SA, Kruzel ML. Lactoferrin as a natural immune modulator. Curr Pharm Des 2009;15:1956-73.

56. Buccigrossi V, de Marco G, Bruzzese E, et al. Lactoferrin induces concentration-dependent functional modulation of intestinal proliferation and differentiation. Pediatr Res 2007;61:410-4.

(English Language Editors: G. Stone and J. Chapnick) 